\title{
BER-Aware Reflecting Elements Allocation for RIS-aided NOMA Networks over Ricean Fading
}

This paper was downloaded from TechRxiv (https://www.techrxiv.org).

LICENSE

CC BY 4.0

SUBMISSION DATE / POSTED DATE

05-01-2022 / 07-01-2022

\section{CITATION}

Asmoro, Krisma; Ramatryana, I Nyoman Apraz; Shin, Soo Young (2022): BER-Aware Reflecting Elements Allocation for RIS-aided NOMA Networks over Ricean Fading. TechRxiv. Preprint. https://doi.org/10.36227/techrxiv.17869058.v1

$\mathrm{DOI}$

10.36227/techrxiv.17869058.v1 


\title{
BER-Aware Reflecting Elements Allocation for RIS-aided NOMA Networks over Ricean Fading
}

\author{
Krisma Asmoro, I Nyoman Apraz Ramatryana, Student Member, IEEE, \\ and Soo Young Shin, Senior Member, IEEE
}

\begin{abstract}
Reconfigurable intelligent surface (RIS) as a supportive technology for aiding downlink non-orthogonal multiple access (NOMA) can enhance the bit error rate (BER) performance. In this paper, a novel BER-aware reflecting elements allocation (REA) on an RIS is proposed to maintain the BER order among paired RIS-NOMA users. The RIS REA is useful for minimizing the average user BER, ompared with a system that allocates the same number of elements to all users. Additionally, the Ricean fading is considered instead of Rayleigh fading as it is more practical and general. Furthermore,an REA optimization objective function for equalizing the user BER is proposed. In order to solve the problem, a modified exhaustive search is proposed to reduce complexity. The distribution of the objective function is observed first; subsequently, the exhaustive search range is determined. Both the analytical and simulation results show that the proposed algorithm can minimize the average user BER.
\end{abstract}

Index Terms-Bit error rate (BER), non-orthogonal multiple access (NOMA), reconfigurable intelligent surface (RIS), Ricean fading, reflecting element allocation (REA)

\section{INTRODUCTION}

$\mathbf{R}$ ECONFIGURABLE intelligent surfaces (RISs) is recently gaining a popularity as a technology to aid wireless technology for 5G and beyond, because of the capability to manipulate signal propagation for both downlink and uplink transmission. An RIS is defined as a planar surface comprising low-cost reflective elements that can work independently [1]. In [2], it is reported that each element can modify channel phase with a several tuning methods. Moreover, in [3] it is reported that an RIS performs better than relaying in term of spectral efficiency and deployment cost. Therefore, due to its numerous advantages, RIS has been integrated with many existing wireless technology as a primary transmitter, an access point (AP) or extending a network coverage, a dual hop communication (DH).

A diverse integration of RIS for aiding wireless telecommunications was performed, in particular, RIS-aided nonorthogonal multiple access. RIS-NOMA is recently gaining focus due to higher channel capacity and low error rates [4], [5]. NOMA is considered as a promising technologies for $5 \mathrm{G}$ and beyond that can fulfill the demands of high data rates, spectral efficiency and massive users connectivity [6]. This is due to the principal work of NOMA which allows user to

This work was supported by the National Research Foundation of Korea (NRF) grant funded by the Korea government (MEST) (No. 2019R1A2C1089542).

The authors are with the WENS Laboratory, Department of IT Convergence Engineering, Kumoh National Institute of Technology, Gumi, South Korea (e-mail: krisma@kumoh.ac.kr, ramatryana@kumoh.ac.kr, wdragon@kumoh.ac.kr). share a same time or frequency resources [7]. The principal work of power domain NOMA (PD-NOMA) is allocating the higher power allocation (PA) for adverse channel condition of cell edge users (CEU), while a cell center user (CCU) perform a successive interference cancellation (SIC) technique to retrieve information [8]. Compare to its predecessor, orthogonal multiple access (OMA), NOMA gives a better performance in a point of view of the ergodic capacity and outage probability for uplink [9] or downlink communication [10], [11].

\section{A. Related Works}

In NOMA, a tradeoff exists between high data rate and spectral efficiency, which results in a relatively high BER. This is because the CCU must perform SIC using the incoming superposition-coded (SC) signal. Therefore, the RIS facilitates the NOMA network because it improves the signal-to-noise ratio (SNR) with signal diversity. Recently, the integration RIS for assisting NOMA has become a topic of considerable research interest, in particular for assisting PD-NOMA. In [5], RIS single-input single-output (SISO) NOMA was proposed, as well as RIS passive beamforming for improving spectral efficiency. In [12], the joint optimization of active beamforming at the base station (BS) and passive beamforming at the RIS was proposed to maximize the signal-to-interferenceplus-noise ratio (SINR) for all users. Therefore, the desired rate performance and user fairness can be achieved. In [13], a downlink PD-NOMA with RIS was proposed to enhance energy efficiency. It can be achieved through passive beamforming design and joint user clustering. In [14], a simple RISNOMA downlink scenario for multiple-input single-output (MISO) BS. It was presumed that the CCU had a line of sight (LoS) to the BS and multiplexed with spatial division multiple access (SDMA). At the same time, CEU had a no LoS to the BS and supported with RISs. In [15], a deployment and passive beamforming design for RIS aided NOMA with MISO BS. The main objective was to maximize energy efficiency and subjects to user data rate requirements. The joint optimization for the active beamforming of MISO-NOMA system was extended in [16], where the main objective was to reduce the total transmit power for multi-cluster user. In [17], proposed a passive beamforming desgin for RIS aided MISO-NOMA system. The idea was to optimize a beamforming weights in order to minimize intercluster interference. In [18], a combination of joint transmission coordinated multipoint (JT-CoMP) on an RIS was proposed to provide more CEU ergodic capacity without sacrificing CEU performane. In [19], it was examined the RIS phase shifter design, between the coherent discrete 
and the random discrete comprehensively. In [20], a capacity fairnes performance of RIS SISO NOMA and RIS MIMO NOMA over Ricean fading channel was investigated. In [21], the symmetrical splitting of RIS elements for downlink CCUs and CEUs was proposed, and it was shown that RIS improved the BER performances of both CCUs and CEUs. In [22] proposed a joint optimization solution for resource allocation problem in RIS-NOMA system. Here, in order to maximize the system throughput, they optimize several aspects, including the channel assignments, power allocation, decoding order, and RIS reflection coefficient. Finally, in [23] considered a RISNOMA downlink scenario with element partitioning, aiming for user fairness. The proposed partitioning could enhance the outage probability and ergodic capacity.

\section{B. Motivation and Contributions}

The physical resource allocation scheme used in this paper is based on a previous works. It can be summarized as follows: The BS transmits a single SC signal to users via an RIS reflection (dual-hop communication), assuming all user positions are within the RIS coverage. The RIS phase and reflection coefficient are adjusted to jointly serve all users. In the proposed scheme, not all users will have the same distance to the BS and RIS. Therefore, physical resource allocation must not be equal among users, because every user has it own demands.

Inspired by [24], we propose a novel reflecting element allocation (REA) for RIS assisted NOMA systems and an efficient physical resource allocation. The main purpose of REA is to determine a suitable ratio of RIS to obtain equivalent BER of NOMA users without adjusting the PA. The RIS serves all NOMA users with different number ratio of elements for each users. The REA is beneficial to the RIS-NOMA systems because it provides a lower average users BER than fairly allocate an RIS elements for all users. The main contributions of this paper can be written as follows:

- The formulation of searching the suitable ratio of REA is proposed in present work, in addition to the result of the suitable ratio.

- In contrast to the conventional exhaustive search, we first examine and predict the distribution of the objective function based on a specified parameter. Furthermore, the range of exhaustive search can be narrowed to decrease system complexity.

- An exact analytical BER RIS-NOMA over DH Ricean fading channel is derived in this works, and the simulation and analytical results presented.

The rest of the paper is organized as follows: In Section II, the proposed system model is described in addition to the relevant mathematical expressions. The analytical or theoretical of BER RIS-NOMA over DH Ricean fading is derived in Section III-A. The problem formulation, solution, and the modified exhaustive search are described comprehensively in in Section III-B and Section III-C. Results of computer simulations are provided in Section IV, followed by the conclusions in Section $\mathrm{V}$.

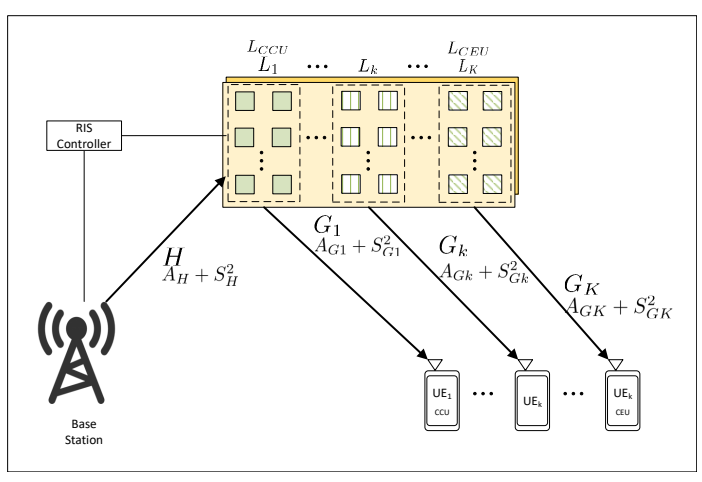

Figure 1. The overall system model of BER-aware REA RIS aided NOMA system

The notation of this paper is written as follows: ${ }_{p} \mathcal{F}_{q}(; ;)$ denotes a generalized hypergeometric function. ${ }_{1} \mathcal{F}_{1}(; ;)$ denotes the confluent hypergeometric function of a first kind. $\mathcal{L}_{n}(\cdot)$ denotes a Laguerre Polynomial with $n$ parameter. The $|\cdot|^{2}$ operator produces a scalar value and denotes the magnitude function of the complex vector. $\lceil\cdot\rceil$ and $\lfloor\cdot\rfloor$ represent ceiling and floor function, respectively. $\mathbb{C}^{a \times b}$ denotes complex-value array with length $a$ and width $b . \operatorname{diag}\{\cdot\}^{d}$ denotes a diagonal matrix with length and width $d . \mathcal{N}\left(A, \sigma^{2}\right)$ denotes a variable distribution with mean $A$ and variance $\sigma^{2} . I_{0}(\cdot)$ is the zeroorder modified Bessel Function of the first kind. $Q_{N}(\cdot, \cdot)$ is the generalized marcum function with $\mathrm{N}$ parameter

\section{System Model}

Based on [23] where $N$ RIS elements split among NOMA user and the incoming NOMA signal is reflected to corresponding user. In this paper, we denotes reflecting element allocation $L_{k}$ and prior to [20], a PD-NOMA downlink scheme is considered with single antenna equipped at base station. The initialization step from Fig 1 is that all the user symbols are modulated and mapped with an M-ary constellation which is denoted by $S_{k}$. Afterward, all of the symbols were multiplied with each user PA coefficient. Additionally, it is assumed that the value of PA is in equivalent to $0<\alpha_{k}<1$. Therefore, the superposition coding for this scenario can be expressed as $\chi=\sum_{k=1}^{K} \sqrt{\alpha_{k} P} S_{k}$, where $P$ is the sum of transmitted power of the BS and $l$ indicates an index of the $k^{t h}$ user from the total of $K$ user. The rule of PA for $K$ user is denoted by $\alpha_{1}<\cdots<\alpha_{k}<\cdots<\alpha_{K}$ where user with $k=1$ always had a better channel condition than the others.

In this paper, we considered a $\mathrm{DH}$ ricean fading scenario for two user NOMA, namely cell edge user (CEU) and cell center user (CCU). The single-hop ricean fading with the presence of line of sight (LOS) and non line of sight (NLOS) path is represented as [25], [26] :

$$
h(t)=\underbrace{\sqrt{\frac{\nu \rho}{\nu+1}}}_{\mathrm{A}:=} e^{\left(j 2 \pi f_{D} \cos (\theta) t+\phi\right)}+\underbrace{\sqrt{\frac{\rho}{\nu+1}}}_{\mathrm{S}:=} g(t),
$$

where $\theta$ is angle of arrival (AoA) at the reciver, $\phi$ the channel phase, $g(t)$ is a narrowband complex Gaussian process, and 
$f_{D}$ is a maximum Doppler Frequency. $\nu=\frac{A^{2}}{S^{2}}$ is the Ricean factor, which consists of the power ratio between LOS component $\left(A^{2}\right)$ and scattered component $\left(S^{2}\right)$. Here, $\rho$ denotes the sum of total received signal power from both LOS and NLOS power components, which is expressed as $\rho=A^{2}+S^{2}$. Based on [27], we define the channel fading matrix contains Gaussian random variables (RV) with non-zero mean $A$ and standard deviation $S$ as follows:

$$
A=\sqrt{\frac{\nu}{2(\nu+1)}}, \quad S=\sqrt{\frac{\nu}{2(\nu+1)}} .
$$

Bassed on Eq. (2), we can infer that $\nu=0$ is the Raylegih fading channel (pure scattered components) and $\nu=\infty$ is the LOS fading channel. The received signal of the $k^{t h}$ user of the proposed system is written as:

$$
y_{k}=H G_{k} \Phi \chi+n_{k},
$$

where $\left[H \in \mathbb{C}^{L_{k} \times 1}, G_{k} \in \mathbb{C}^{L_{k} \times 1}\right]$ is a Ricean distributed fading channel $\mathcal{N}\left(A_{H}, S_{H}^{2}\right)$ within BS to RIS element and $\mathcal{N}\left(A_{G}, S_{G}^{2}\right)$ between RIS elements to $k^{t h}$ NOMA users, respectively.In addition, the $\nu$ factor for $H$ and $G$ are denoted with $\nu_{H}$ an $\nu_{G}$, respectively. The $\Phi^{L_{k}}$ denotes a phase shift response [14] with ON/OFF switch and $\Phi^{L_{k}} \in$ $\operatorname{diag}^{L_{k}}\left\{e^{j \phi_{1}}, \cdots, e^{j \phi_{i}}, \cdots, e^{j \phi_{L_{k}}}\right\}$. Here, $e^{j \phi_{i}}$ denotes the response phase of $i^{t h}$ elements, which minimizes the channel phases, and $n_{k}$ is an Additive White Gaussian Noise (AWGN) of user $k$ with $\mathcal{N}(0,1)$.

The capability of each $i^{\text {th }}$ RIS element that can be easily turned ON and OFF, leads to the asymmetric element allocation for $k^{t h}$ NOMA users. Thus, the number of serials $i$ elements that have to be activated to assist downlink communications denoted by $L_{k}$, and the elegant equation of the total of RIS elements is $N=\sum_{k=1}^{K} L_{k}$ where $L_{1}<\cdots<L_{K}$ is due to the lower value of $\nu$ that $K^{\text {th }}$ user had. All users had the channel statistic information (CSI) on the receiver side, and $K-1$ user were engaging perfect SIC to decode its data.

\section{Proposed BER-AwARE REA}

Throughout this section, the derivation for calculating the theoretical average bit error rate (BER) of user 1 and 2 RIS NOMA by exploiting the moment generating function (MGF) equation [28], [29]. On top of that, the problem formulation and solution approach of RIS EA in order to equalize the BER among NOMA users or minimizing BER for $k>1$ NOMA users. Additionally, contrary to the previous work [21], here the QPSK modulation $(M=4)$ was employed for $\mathrm{CCU}$ and $\mathrm{CEU}$ for the sake of fair problem formulation.

\section{A. RIS NOMA exact Error Probability}

Suppose the channel magnitudes and phases are approximately denoted by $\alpha_{i} e^{j \psi_{i}}$ and $\beta_{i, l} e^{j \theta_{i, k}}$ for the channel $h_{i}$, $g_{i, k}$ respectively. Here, the effect of $e^{j \phi_{i}}$ is to manipulating the EM wave and maximize SNR by eliminating all the channel phases, hence the value of RIS phase response written as $\phi_{i}=\psi_{i}+\theta_{i, k}$. Then, the instantaneous SINR in the OMA receiver is formulated as:

$$
\gamma=\frac{P\left|\sum_{i=1}^{N} \alpha_{i} \beta_{i, k} e^{j\left(\phi_{i}-\theta_{i}-\psi_{i, k}\right)}\right|^{2}}{N_{0}}=\frac{P\left|\sum_{i=1}^{N} \alpha_{i} \beta_{i, k}\right|^{2}}{N_{0}}
$$

where $P$ is a transmit power and $N_{0}$ is the noise power within th AWGN distribution. On the other hand, contrary to the OMA system, the SNR for the proposed RIS-NOMA system for two users is affected by inter-user interference. Thus it can be written as follows:

$$
\begin{aligned}
\gamma_{1} & =\frac{\sqrt{\alpha_{1} P}\left|\sum_{i=1}^{L_{1}} \alpha_{i} \beta_{i, 1}\right|^{2}}{N_{0}}, \\
\gamma_{2} & =\frac{\sqrt{\left(1-\alpha_{1}\right) P}\left|\sum_{i=L_{1}+1}^{L_{2}} \alpha_{i} \beta_{i, 2}\right|^{2}}{N_{0}+\sqrt{\alpha_{1} P}\left|\sum_{i=L_{1}+1}^{L_{2}} \alpha_{i} \beta_{i, 2}\right|^{2}+1},
\end{aligned}
$$

where $1-\alpha_{1}$ is the PA of user 2. Considering $\alpha_{i}$ and $\beta_{i, l}$ are the independent ricean random variables. Mean and variance of proposed system was derived and proved by Theorem and Lemma bellow.

Theorem 1 (Sum of Ricean Variables theorem). Let $R_{i}$ $\mathcal{N}\left(A_{i}, \sigma^{2}\right), i=1, \cdots, N$ be a set of Ricean random variables. The summation of Ricean Random Variables is denoted as [30] [31]:

$$
S R=\sum_{i=1}^{N} R_{i}^{2}\left(A_{i}, \sigma^{2}\right) \sim \mathrm{nc}-\chi_{2 N}^{2}\left(\frac{x}{\sigma^{2}}, \frac{A_{N}^{2}}{\sigma^{2}}\right)
$$

with $\chi_{2 N}^{2} a \mathrm{nc}-\chi^{2}$ with $2 N$ degrees of freedom and $A_{N}^{2}=$ $A_{N}^{2}=\sum_{i}\left|A_{i}\right|^{2}$.

Refer to the theorem 1 is the statement in the next lemma.

Lemma 1. Define two independent ricean random variables $\alpha_{i}$ and $\beta_{i, k}$ respectively. The mean and variance for $N$ element RIS are denoted as follows:

$$
\begin{gathered}
E\left[\alpha_{i} \beta_{i, l}\right]=\mu_{1}=\mu=N \sqrt{\frac{\pi}{2}} S \mathcal{L}_{1 / 2}(-\nu) \\
V A R\left[\alpha_{l, i} \beta_{i}\right]=\sigma^{2}=N\left[A^{2}+S^{2}-\frac{\pi S^{2}}{2} \mathcal{L}_{1 / 2}^{2}(-\nu)\right] .
\end{gathered}
$$

$\mu$ is the first raw moments of $\mu_{k}^{\prime}=\sigma^{k} 2^{k / 2} \Gamma(1+$ $k / 2) L_{k / 2}(-\nu)$ and $\sigma^{2}$ is the second central moments of $\mu_{k}^{\prime}$ 32].

Proof. To prove it, we refer to the closed form approximations to Ricean sum distribution proposed by J.Hu at all [30]. First, define probability density function (PDF) for Ricean fading with parameter $A$ (non centrality parameter) and $S$ (Gaussian scatter components) from 2

$$
f_{R_{i}}\left(x_{i}\right)=\frac{x_{i}}{S_{i}^{2}} \exp \left(-\frac{x_{i}^{2}+A_{i}^{2}}{2 S_{i}^{2}}\right) I_{0}\left(\frac{x_{i} A_{i}}{S_{i}^{2}}\right), \quad x_{i} \geq 0,
$$


where $x_{i}$ is the amplitude of $N$ RIS elements independent RV's. Then, we integrate $\nu$ factor, the PDF becomes:

$$
\begin{aligned}
f_{R_{i}}\left(x_{i}\right)=\frac{2 x_{i}\left(\nu_{i}+1\right)}{\rho_{i}} & \exp \left(-\nu_{i}-\frac{\left(\nu_{i}+1\right) x_{i}^{2}}{\rho_{i}}\right) \\
& \times I_{0}\left(2 x_{i} \sqrt{\frac{\nu_{i}\left(\nu_{i}+1\right)}{\rho_{i}}}\right) .
\end{aligned}
$$

The envelope of the signal component at combiner output of an equal gain combining (EGC) [33] is defined as $Z=\sum_{i=1}^{N} R_{i}$. The RV $S$ in Eq. (6), has a noncentral chi-square distribution with $2 N$ degrees of freedom. Therefore, with the expressed PDF condition in Eq. (9) and [28], the PDP of $S R$ denotes by:

$$
\begin{array}{r}
f_{S R}(x)=\frac{1}{2 S^{2}}\left(\frac{x}{A^{2}}\right)^{\frac{N-1}{2}} \exp \left(-\frac{x+A^{2}}{2 S^{2}}\right) \\
\quad \times I_{N-1}\left(\frac{\sqrt{x} A}{S^{2}}\right), \quad x \geq 0,
\end{array}
$$

where $A^{2}=\sum_{i=1}^{N} A_{i}^{2}$ and $I_{N-1}(\cdot)$ is the $(\mathrm{N}-1)^{t h}$ order modified Bessel Function of the first kind. Obtain a CDF by integrating Eq. (11) and resulting in:

$$
F_{S R}(x)=1-Q_{N}\left(\frac{A}{S}, \frac{\sqrt{x}}{S}\right),
$$

where RV $S R$ represents a envelope of the signal component at the combiner output of a MRC system in Ricean fading [34]. Thus the approximation for the CDF of RV $S R$, in respect to $N$ elements is defined as:

$$
\begin{aligned}
F_{N} & =1-Q_{N}\left(\frac{b}{c_{1}}, \frac{t}{c_{2}}\right), \\
b & =\sqrt{\frac{N \rho \nu}{\nu+1}}
\end{aligned}
$$

where $c_{1}$ and $c_{2}$ are the constants, optimally obtained from [35] and $t$ contains a normalized arguments, defined as $\frac{x}{\sqrt{N}}$. Finally, the PDF of $S R$ was obtained by first derivative order of CDF Eq. 13 and it is expressed as follows:

$$
\begin{aligned}
f_{N} \triangleq \frac{\mathrm{d} F_{N}(t)}{\mathrm{d} t}=\frac{t^{N}}{c_{2}^{2}}\left(\frac{c_{1}}{c_{2} b}\right)^{N-1} \exp & \left(-\frac{t^{2}}{2 c_{2}^{2}}-\frac{b^{2}}{2 c_{1}^{2}}\right) \\
& \times I_{N-1}\left(\frac{t b}{c_{1} c_{2}}\right) .
\end{aligned}
$$

Based on proposed CDF and PDF as denoted in Eq. (13) and Eq. (14) respectively, we can conclude that the number of $N$ is impacting the skewness of both density function. However, to make the analytical approach and simulation result is match, we follow a central limit theorm where the number $N$ is far greater than $1(N>>1)$. Additionally, with exact PDF approximation based on $\mathrm{CDF}$, we can prove that number of elements are affecting mean and variance as mentioned in Eq. (7) and Eq. (8), respectively.
Lemma 2. The total of channel power $\rho$ for dual hop Ricean fading channel is represented as:

$$
\rho=A^{2}+A^{2}+2 S^{2}
$$

Proof. Fading channel $\mathrm{H}$ and $\mathrm{G}$ are consist of complex numbers, and both of it are Independent and identically distributed (IID) RV with mean $\left[A_{H}, A_{G}\right]$ respectively, and standard deviation $S$. Here, to prove it, we utilize the ricean RV identity as follows :

$$
\begin{aligned}
V & \triangleq \sqrt{H^{2}+G^{2}} \\
E\left\{H^{2}\right\} & =S^{2}+A_{H}^{2} \\
E\left\{G^{2}\right\} & =S^{2}+A_{G}^{2} \\
E\left\{\left(H-A_{H}\right)^{2}\right\} & =E\left\{\left(G-A_{G}\right)^{2}\right\}=S^{2} \\
E\left\{V^{2}\right\} & =E\left\{H^{2}\right\}+E\left\{G^{2}\right\} \\
E\left\{V^{2}\right\} & =2 S^{2}+A_{H}^{2}+A_{G}^{2} .
\end{aligned}
$$

Here, it was predefined that $\left[\alpha_{i}, \beta_{i, l}\right]$ were non-central chi square RV with a single degree of freedom (DoF) [28], therefore, $M G F(\omega)=\left(\frac{1}{\sqrt{\left.1-2 \sigma^{2} \omega\right)}}\right) \exp \left(\frac{\mu^{2} \omega}{\left.1-2 \sigma^{2} \omega\right)}\right)$ whereas $\omega$ denoting the $\omega^{t h}$ moment of MGF. With the derived $\left[\mu, \sigma^{2}\right]$, thus the formulation of MGF with a certain $P / N_{0}$ is written as:

$$
\begin{array}{r}
M G F(\omega)=\sqrt{\frac{1}{1-\frac{\omega N\left[A^{2}+S^{2}-\frac{\pi S^{2}}{2} \mathcal{L}_{1 / 2}^{2}(-\nu)\right] \rho P}{N_{0}}}} \\
\quad \exp \left(\frac{\frac{\omega N^{2} \pi S^{2} \mathcal{L}_{1 / 2}^{2}(-\nu)}{2} \frac{\rho P}{N_{0}}}{1-\frac{\omega N\left[A^{2}+S^{2}-\frac{\pi S^{2}}{2} \mathcal{L}_{1 / 2}^{2}(-\nu)\right] \rho P}{N_{0}}}\right) .
\end{array}
$$

Since the proposed system is utilizing $M$-ary Modulation and the emitted signal transmitted through the Rayleigh fading channel, suppose $M$-PSK modulation. The MGF for analytical symbol error rate (SER) becomes [36]:

$$
P e_{\gamma s}=\frac{1}{\pi} \int_{0}^{\frac{(M-1) \pi}{M}} M G F_{\gamma_{s}}\left(-\frac{\sin (\pi / M)}{\sin ^{2} \phi}\right) d \phi .
$$

Therefore, by operating substitution (17) and 18, the SER for RIS aided OMA system is expressed as:

$\overline{P_{e}}=\frac{1}{\pi} \int_{0}^{\frac{(M-1) \pi}{M}}\left(\frac{1}{1+\frac{N\left[A^{2}+S^{2}-\frac{\pi S^{2}}{2} \mathcal{L}_{1 / 2}^{2}(-\nu)\right]^{2} \sin (\pi / M)}{\sin ^{2} \xi} \frac{\rho P}{N_{0}}}\right)$

$$
\exp \left(\frac{-\frac{N^{2} \pi S^{2} \mathcal{L}_{1 / 2}^{2}(-\nu) \sin (\pi / M)}{2 \sin ^{2} \xi} \frac{\rho P}{N_{0}}}{1+\frac{N\left[A^{2}+S^{2}-\frac{\pi S^{2}}{2} \mathcal{L}_{1 / 2}^{2}(-\nu)\right]^{2} \sin (\pi / M)}{\sin ^{2} \xi} \frac{\rho P}{N_{0}}}\right) d \xi
$$

Further, for deriving analytical SER of RIS-NOMA user 2 where the user is treating $y_{1}$ as noise (5) and implementing ML detector to detect its symbol is initialized by determining the decision boundary [29]. According to ML decision boundary 
with QPSK modulation, user 2 decode symbol in signal constellation (SC) IQ plane within boundaries $\Re\left\{y_{2}\right\}<0$ or $\Re\left\{y_{2}\right\} \geq 0$ and $\Im\left\{y_{2}\right\}<0$ or $\Im\left\{y_{2}\right\} \geq 0$, where $\Re\{\cdot\} \Im\{\cdot\}$ denoting in-phase and quadrature component of $\mathrm{SC}$ received signal $y_{2}$ respectively. Therefore, the bit error probability (BEP) for the in-phase component of SC in Qfunction form is denoted by:

$$
P_{e 2, \Re}=\frac{1}{2}\left[Q\left(\sqrt{\Upsilon_{a}^{2} \zeta_{2}}\right)+Q\left(\sqrt{\Upsilon_{b}^{2} \zeta_{2}}\right)\right],
$$

with $\Upsilon_{a}=\sqrt{\varepsilon_{2}}+\sqrt{\varepsilon_{1}} ; \Upsilon_{b}=\sqrt{\varepsilon_{2}}-\sqrt{\varepsilon_{1}}$.

Further, $\Upsilon$ is denoting the baseband SC symbols for users, $\varepsilon_{2}$ is indicating user 2 PA $\sqrt{P\left(1-\alpha_{1}\right)}$ while $\varepsilon_{1}$ denoting user 1 PA $\sqrt{P \alpha_{1}} . Q(\cdot)$ is representing the Gaussian error performance $Q(x)=(1 / \sqrt{2 \pi}) \int_{x}^{\infty} \exp \left(-u^{2} / 2\right) d u$. Additionally, $\zeta_{2}$ represents the user 2 channel gain and noise power $\frac{\rho_{2}}{N_{0}}$ since the received signal $y_{2}$. Similarly, with the in-phase component of $\mathrm{SC}$, the BEP for SC of quadrature component had the same formulation with 200 . Therefore $P_{e 2}=\left(P_{e 2, \Re}+P_{e 2, \Im}\right) / 2$. At this point, it is clear that the BEP of user 2 is multiplied by 0.5 due to the symmetrical QPSK SC symbols. Finally, the exact SER for user 2 based on BEP (20) is formulated as in (21), where $A\left(L_{2}\right)=\sigma^{2} \sin (\pi / M), B\left(L_{2}\right)=-\mu^{2} \sin (\pi / M)$ and $A\left(L_{l}\right)$ and $B\left(L_{l}\right)$ denoting the multiplication between $\omega^{\text {th }}$ moment of MGF with $\mu$ and $\sigma^{2}$ in Eq. (7) and Eq. (8), respectively.

However, the BEP of user 1 where the user is performing SIC was defined as $P_{e 1}=P_{e 1}\left(e \mid\right.$ correct $\left.b_{2}\right)+P_{e 1}(e \mid$ error $\left.b_{2}\right)$. In other words, the $P_{e 1}$ is obtained by adding the BEP under condition $b_{2}$ (bit user 2) detected correctly and erroneously. Thus, in the form of Q-function, the BEP of the bit that detected correctly at the in-phase component SC is:

$$
P_{e 1, \Re}\left(e \mid \text { correct } b_{2}\right)=\frac{1}{2}\left[2 Q\left(\sqrt{\left.\varepsilon_{1} \zeta_{1}\right)}-Q\left(\sqrt{\Upsilon_{a}^{2} \zeta_{1}}\right)\right]\right. \text {, }
$$

where $\zeta_{1}$ is the notation of the user channel $1 \frac{\rho_{1}}{N_{0}}$. In a different manner with the BEP under condition first bit detected correctly, then for the condition when $b_{2}$ detected erroneously is expressed as:

$$
\begin{array}{r}
P_{e 1, \Re}\left(e \mid \text { error } b_{2}\right)=\frac{1}{2}\left[Q\left(\sqrt{\Upsilon_{b}^{2} \zeta_{1}}\right)-\right. \\
\left.Q\left(\sqrt{\Upsilon_{c}^{2} \zeta_{1}}\right)+Q\left(\sqrt{\Upsilon_{d}^{2} \zeta_{1}}\right)\right],
\end{array}
$$

with $\Upsilon_{c}=2 \sqrt{\varepsilon_{2}}-\sqrt{\varepsilon_{1}} ; \Upsilon_{d}=2 \sqrt{\varepsilon_{2}}+\sqrt{\varepsilon_{1}}$.

Similarly, for calculating BEP at SC $\Im$ component by following (23) and 24) thoroughly. Additionally, it is identical with the calculation of $P_{e 2}, 0.5$ is attached to the $P_{e 1, \Re}$ to calculate the average BEP of both in-phase and quadrature SC, hence SER calculation for user 1 is formulated in 22 .

\section{B. Problem Formulation}

The principal work of asymmetrical REA for RIS NOMA users is aiming at the same order of SER among downlink users. In simple words, the proposed system attempts to choose the ideal amount of elements allocation for each users until

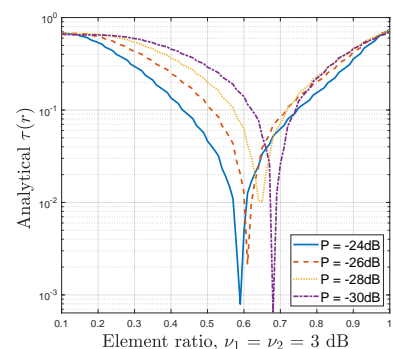

(a)

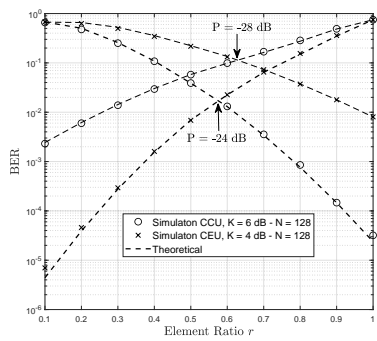

(b)
Figure 2. (a)The result of $\tau(r)$ distribution for different transmit power and element ratio. (b) The simulation and analytical result of equilibrium point (pointed by arrow) with $d=0.1$

it had an approximately similar SER $P_{e 2} \approx P_{e 1}$. Thus, in the case of two users SER value is modelled as $\left|P_{e 2}-P_{e 1}\right|<$ 0.1 . Then, the optimization problem of REA RIS-NOMA with finite $N$ is defined as:

$$
\begin{aligned}
\underset{\left\{\alpha_{1}, N, \Omega_{l}\right\}}{\text { Minimize }} & \tau=\left|\overline{P_{e 2}}\left(L_{2}\right)-\overline{P_{e 1}}\left(L_{1}\right)\right| \\
\text { Subject to } & \tau<0.1, \tau=\left(10^{-2}, 10^{-3}\right], \\
& e^{j \phi_{i}}=(0,2 \pi], \\
& L_{1} \neq L_{2}, L_{1}<L_{2}, N=L_{1}+L_{2}, \\
& 0 \leq \alpha_{1} \leq 0.5, \alpha_{2}=1-\alpha_{1} .
\end{aligned}
$$

where $\tau$ is the difference between SER user 1 and 2, moreover, the proposed system is aiming the high similarity for both user $(\tau<0.1)$ therefore the $\tau$ value is in between $10^{-2} \leq \tau \leq$ $10^{-3}$. The other important thing to notice that these problem formulation constraints is the fixed PA for both users $\left(\alpha_{1}\right)$. $e^{j \phi_{i}}$ denoting the discrete RIS response phase of $i^{t h}$ element [37].

\section{Problem solution}

In order to solve Eq. (25), we define $\tau$ as an objective function, $\tau(r)$ as a scalar value of objective function with parameter $r$, and $S$ as a feasible value of interest of $r$. However, solving the problem with the conventional exhaustive search Optimization of number element has previously proposed in [38] where the monotonically increasing number of elements resulting in unimodality of energy consumption. Therefore, we inspect first the distribution of objective function $\tau(r)$ with ratio test $r \in[0.1,0.1+1 d, 0.1+2 d, \cdots, 1]$, where $d=0.01$ as a interval. Fig. 2(a) shows that the distribution of $\tau(r)$ is decreasing first then increasing again after one point reached. The same behaviour was shown for different $P$ where $P \in[-24,-26,28,-30]$. Fig. 2 2 b) is pointing out that by using the same $P$ and $N$ for both user will be resulting in equilibrium point for a certain value of $r$ (pointed by arrow). Therefore, based on the figure, we define that $\tau(r)$ is unimodal and the definition is written as follows:

Definition 1 (Unimodal Function). A function $\tau(r)$ is unimodal on the interval $r-d \leq r \leq r+d$ if and only if it is 


$$
\begin{aligned}
& \overline{P_{e 2}}=\frac{1}{2 \pi} \int_{0}^{\frac{3 \pi}{4}}\left(\frac{1}{1+\frac{A\left(L_{2}\right) \Upsilon_{a}^{2} \zeta_{2}}{\sin ^{2} \xi}}\right)^{\frac{1}{2}} \exp \left(\frac{\frac{B\left(L_{2}\right) \Upsilon_{a}^{2} \zeta_{2}}{\sin ^{2} \xi}}{1+\frac{A\left(L_{2}\right) \Upsilon_{a}^{2} \zeta_{2}}{\sin ^{2} \xi}}\right)+\left(\frac{1}{1+\frac{A\left(L_{2}\right) \Upsilon_{b}^{2} \zeta_{2}}{\sin ^{2} \xi}}\right)^{\frac{1}{2}} \exp \left(\frac{\frac{B\left(L_{2}\right) \Upsilon_{b}^{2} \zeta_{2}}{\sin ^{2} \xi}}{1+\frac{A\left(L_{2}\right) \Upsilon_{b}^{2} \zeta_{2}}{\sin ^{2} \xi}}\right) d \xi \\
& \overline{P_{e 1}}=\frac{1}{2 \pi} \int_{0}^{\frac{3 \pi}{4}} 2\left(\frac{1}{1+\frac{A\left(L_{1}\right) \varepsilon_{1} \zeta_{1}}{\sin ^{2} \xi}}\right)^{\frac{1}{2}} \exp \left(\frac{\frac{B\left(L_{1}\right) \varepsilon_{1} \zeta_{1}}{\sin ^{2} \xi}}{1+\frac{A\left(L_{1}\right) \varepsilon_{1} \zeta_{1}}{\sin ^{2} \xi}}\right)-\left(\frac{1}{1+\frac{A\left(L_{1}\right) \Upsilon_{a}^{2} \zeta_{1}}{\sin ^{2} \xi}}\right)^{\frac{1}{2}} \exp \left(\frac{\frac{B\left(L_{1}\right) \Upsilon_{a}^{2} \zeta_{1}}{\sin ^{2} \xi}}{1+\frac{A\left(L_{1}\right) \Upsilon_{a}^{2} \zeta_{1}}{\sin ^{2} \xi}}\right)+ \\
& \left(\frac{1}{1+\frac{A\left(L_{1}\right) \Upsilon_{b}^{2} \zeta_{1}}{\sin ^{2} \xi}}\right)^{\frac{1}{2}} \exp \left(\frac{\frac{B\left(L_{1}\right) \Upsilon_{b}^{2} \zeta_{1}}{\sin ^{2} \xi}}{1+\frac{A\left(L_{1}\right) \Upsilon_{b}^{2} \zeta_{1}}{\sin ^{2} \xi}}\right)-\left(\frac{1}{1+\frac{A\left(L_{1}\right) \Upsilon_{c}^{2} \zeta_{1}}{\sin ^{2} \xi}}\right)^{\frac{1}{2}} \exp \left(\frac{\frac{B\left(L_{1}\right) \Upsilon_{c}^{2} \zeta_{1}}{\sin ^{2} \xi}}{1+\frac{A\left(L_{1}\right) \Upsilon_{c}^{2} \zeta_{1}}{\sin ^{2} \xi}}\right)+ \\
& \left(\frac{1}{1+\frac{A\left(L_{1}\right) \Upsilon_{d}^{2} \zeta_{1}}{\sin ^{2} \xi}}\right)^{\frac{1}{2}} \exp \left(\frac{\frac{B\left(L_{1}\right) \Upsilon_{d}^{2} \zeta_{1}}{\sin ^{2} \xi}}{1+\frac{A\left(L_{1}\right) \Upsilon_{d}^{2} \zeta_{1}}{\sin ^{2} \xi}}\right) d \xi
\end{aligned}
$$

monotonic on either side of the single optimal point $r^{*}$ in the interval. In other words, if $r^{*}$ is the single minimum point of $\tau(r)$ in the range $r-d \leq r \leq r+d$, then $\tau(r)$ is unimodal on the interval if and only if for any two points $r_{1}$ and $r_{2}$

$$
\begin{array}{lll}
r^{*} \leq r_{1} \leq r_{2} \quad \text { implies that } \quad & \tau\left(r^{*}\right) \leq \tau\left(r_{1}\right) \leq \tau\left(r_{2}\right) \\
r^{*} \geq r_{1} \geq r_{2} & \text { implies that } \quad \tau\left(r^{*}\right) \leq \tau\left(r_{1}\right) \leq \tau\left(r_{2}\right)
\end{array}
$$

Definition 2 (Global Minimum). A function $\tau(r)$ defined on a set $\mathrm{S}$ attains its global minimum at a point $r^{* *} \in S$ if and only if

$$
\tau\left(r^{* *}\right) \leq \tau(r) \quad \forall r \in S
$$

Definition 3 (Local Minimum). A function $\tau(r)$ defined on $\mathbf{S}$ has a local minimum (relative minimum) at a point $r^{*} \in S$ if and only if there exist an $\varepsilon>0$ such that

$$
\tau\left(r^{*}\right) \leq \tau(r)
$$

for all $r \in S$ satisfying $\left|r-r^{*}\right|<\varepsilon$

Remark. Based on the definition, Algorithm 11 was constructed with remarks as follows:

1) The objective function is unimodal, thus the local minimum automatically becomes the global minimum.

2) Special case with $d<<0.01$, more than one local minimum are possible and the global minimum can only found by locating all local optima and selecting the minimum one, written as:

$$
r^{* *}=\min \left\{\left[r_{1}^{*}, r_{2}^{*}, \cdots, r_{s}^{*}\right] \in S\right\},
$$

where $S$ is a set of local minimum.

3) The unimodality of $\tau(r)$ can decrease the exhaustive search iteration by $50 \%$.

In Algorithm 1 the input is total elements $N, r, d, P, \nu_{k}$ factor for each user and $\alpha$. On this algorithm we following the PD-NOMA rules where user with lower PA (CCU) needs more $N$ than CEU. Therefore, exhaustive search starts from $r=0.5$ and keeps increasing until $r=1$. The initialization part started by calculating REA $L_{1}$ and $L_{2}$, respectively. Then calculate analytical $\tau(r)$ with SER analytical approach from Eq. 21) and Eq. 22), respectively. Afterward, calculate $\tau(r+d)$ and compare it with $\tau(r)$. If the condition is $\tau(r+d)>\tau(r)$ then update $S=\tau(r)$. However, if the condition is $\tau(r+d)<\tau(r)$ then update $S=\tau(r+d)$. Afterward, the proposed system do an iteration with parameter $r$ until $r=1$. Finally, to calculate $r^{* *}$ utilize the equation Eq. 26.

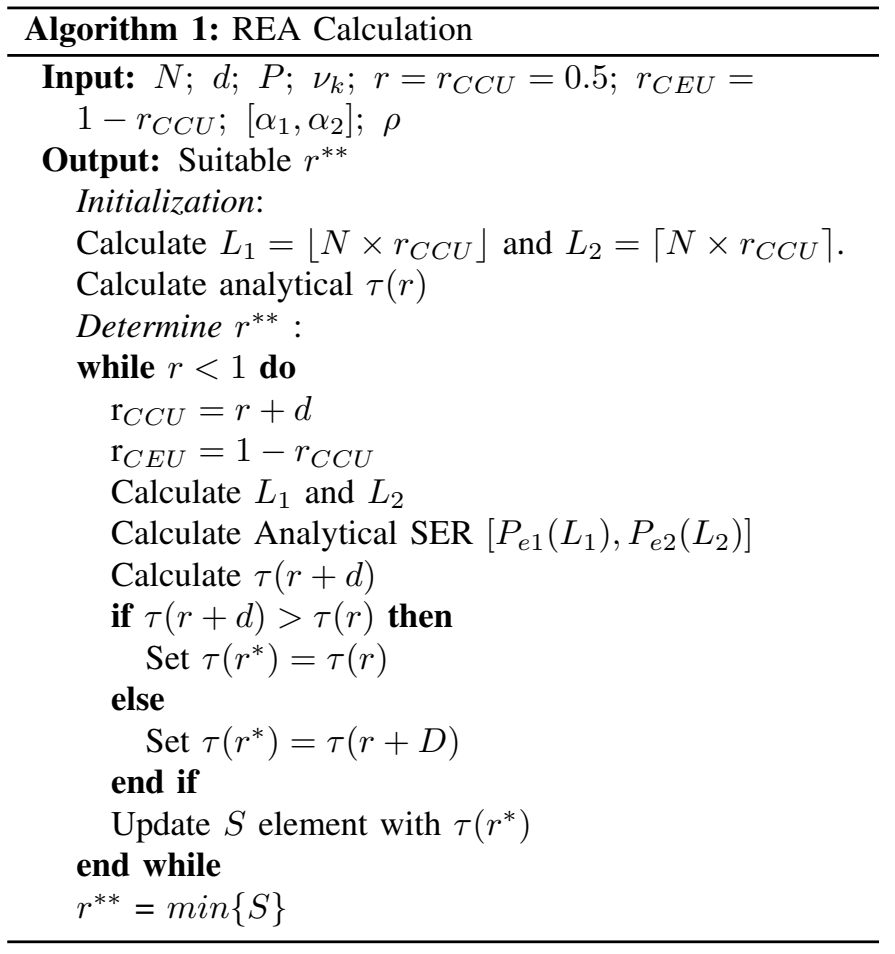

\section{NumericAl Results}

The other parameters which were considered for Algorithm 1 and for the purpose to obtain $r^{* *}$ are $\alpha_{1}=0.3 ; \alpha_{2}=$ $0.7, P \in[-60 \leq \cdots \leq-14] d B, N_{0}=1, d=0.001, \rho$ from Eq. 21, $10^{5}$ monte carlo simulation, $N=128,256,512$. Additionally, $\alpha$ value was determined based on optimal PA for NOMA 2 users [39]. In order to calculate BER, the conversion 
Table I

The RESUlt of Algorithm 1 FOR DiFFERENT $P(d B), N$, AND $\nu_{k}=\left[\nu_{C C U} ; \nu_{C E U}\right]$

\begin{tabular}{rccccccc}
\hline & \multicolumn{2}{c}{$[\nu=8 d B ; 5 d B]$} & & \multicolumn{2}{c}{$\nu=6 d B ; 4 d B]$} \\
\cline { 2 - 4 }$P(\mathrm{~dB})$ & $N=128$ & 256 & 512 & & 128 & 256 & 512 \\
\hline-60 & 0.69 & 0.69 & 0.74 & & 0.69 & 0.7 & 0.73 \\
-56 & 0.69 & 0.72 & 0.79 & & 0.69 & 0.72 & 0.79 \\
-52 & 0.71 & 0.76 & 0.8 & & 0.71 & 0.76 & 0.8 \\
-48 & 0.74 & 0.8 & 0.74 & & 0.74 & 0.81 & 0.75 \\
-44 & 0.79 & 0.77 & 0.68 & & 0.79 & 0.78 & 0.69 \\
-40 & 0.8 & 0.72 & 0.61 & & 0.8 & 0.72 & 0.62 \\
\hline
\end{tabular}

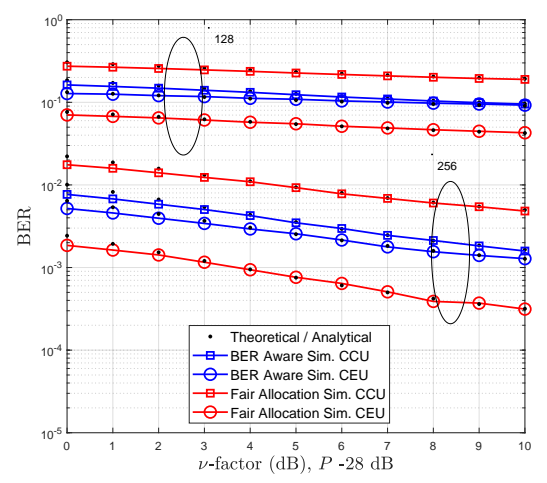

Figure 3. Simulation and Analytical BER result for CCU and CEU user over different value of $\nu$ factor.

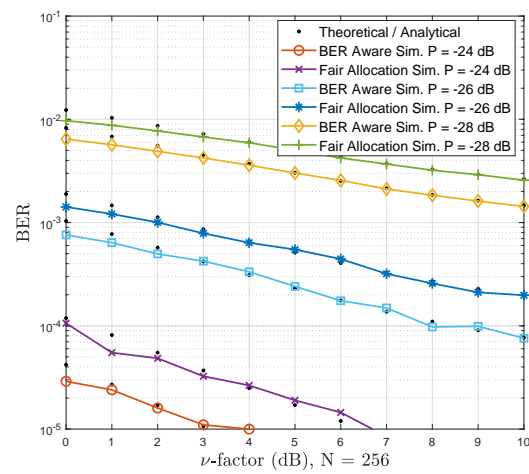

Figure 4. Average user BER result comparison between BER Aware system and fair allocation over different value of $\nu$ factor

is $\approx \overline{P_{e}} / \log _{2}(M)[40]$ and $M=4$ QPSK modulation. The $\nu$ factor for dual hop channel are $\nu_{H}=\nu_{G}=\nu_{k}$.

The result of Algorithm 1 is presented in Table [1 with determined transmit SNR $P$. Here, we can see that the different number of $\nu$ factor of users had, is not giving a significant impact to the result of $r^{* *}$. Therefore, it is feasible to find the suitable ratio with various number of $\nu$ factor. Based on this table, it can be inferred that the $r^{* *}$ is increased to 0.8 to a certain $P$ then decreasing again for higher $P$ value.

Fig. 3 and Fig. 4 show the result of BER with various $\nu_{\text {test }}$ factor where $\nu_{\text {test }}=\nu \in[1,2, \cdots, 10]$. On this figure, we set parameter $\nu_{\text {test }}=\nu_{C E U}=\nu_{C C U}$ for fair comparison. Fig. 3 shows that REA obtained from Algorithm 1 (trained with $\nu_{k}, \nu_{C C U}=6 d B, \nu_{C E U}=4 d B$ is feasible throughout every value of $\nu$. It can be inferred that BER for both user is similar for higher value of $\nu$ factor as well as the analytical and simulation. In this figure, the impact of higher $N$ is resulting in better BER with the same transmit power level $(P=28 d B)$. We also investigate the benefit of BER-aware in Fig. 4 compare to fair elements allocation. Define that $\nu=$ 3 and BER is average users BER mean $\left\{P_{e C C U}, P_{e C E U}\right\}$. With transmit power $(P=-24 d B)$ for proposed system is $6.74 \times 10^{-3}$ whilst the fair allocation is $4.22 \times 10^{-3}$, respectively. $P=-26 d B$ is $7.87 \times 10^{-4}$ and $4.23 \times 10^{-4}$. $P=-28 d B$ is $3.25 \times 10^{-5}$ and $1.1 \times 10^{-5}$.

Fig. 5 shows a result of BER over different transmit SNR $P$ with $r^{* *}$ obtained from Table I] Fig. 5(a) $(\nu=6,4)$ shows the suitable ratio $r$ for transmitting SNR. Targeting the BER $<10^{3}$ for $N=128$ is approximately acquired at $P=-20 \mathrm{~dB}$ while $N=256$ and $N=512$ were $P \approx-24 d B$ and $P \approx-28 d B$ respectively. Thus, it can be concluded that increasing the $N$ elements two times higher than before is lowering the minimum requirement of $P$ by $\approx-4 d B$ in order to achieve the targeted BER. Moreover, in comparison with the proposed system, Fig. 5(b) shows the BER for both users with $r=0.5$ for all $P$. On this figure, with the total available elements $N=128$, to achieve targeted BER for $\mathrm{CCU}$ is improving $\approx 2 d B$ while the trade-off for CEU is the degradation value of $P$ as far as $\approx 3$. Here, it can be seen that the more PA that CEU had, means more degradation of CCU BER. Hence increasing number of RIS elements is required to mitigate the worst channel condition. Fig. 5(c) shows that the system with proposed system BER aware, the average user BER is improving. With the different number of elements $N$ the improvement remains the same. This figure shows that a same number of $N$ and $P$ giving a better performance. The low value of $P$ is not giving a significant improvement for proposed system BER-aware. However, for higher $P$ is giving a better performance. Therefore, define $N=512$ with $P=-30 \mathrm{~dB}$, the error performance for fair $r$ is on ordo $10^{-4}$ while BER-Aware system is $10^{-5}$. This situation also applied for $N=128$ with $P=-18 d B$ and $N=256$ with $P=-24 d B$, respectively.

\section{CONCLUSiOnS}

In this letter, a novel BER-aware reflecting element allocation (REA) in RIS was proposed to maintain the same order of BER among paired RIS-NOMA users. Both analytic and simulation results showed that the proposed algorithm could achieve the same order of BER among multiple RIS-NOMA users. Moreover, it was shown that the proposed algorithm could be applicable to all $\nu$ factor. The proposed algorithm could enhance the average BER among multiple RIS aided NOMA users. Optimal element allocation in uplink RIS aided NOMA network with specific target BER could be one of future works. 


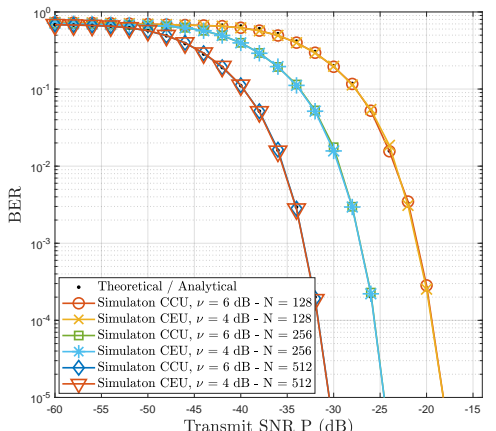

(a)

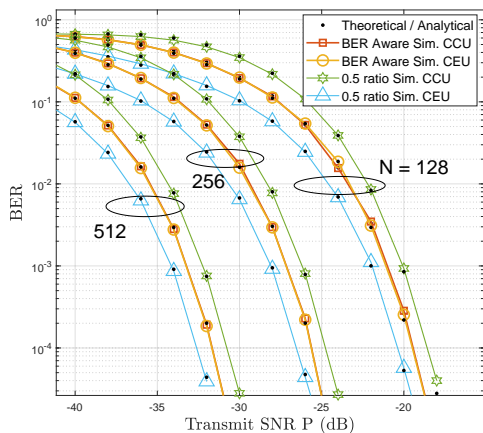

(b)

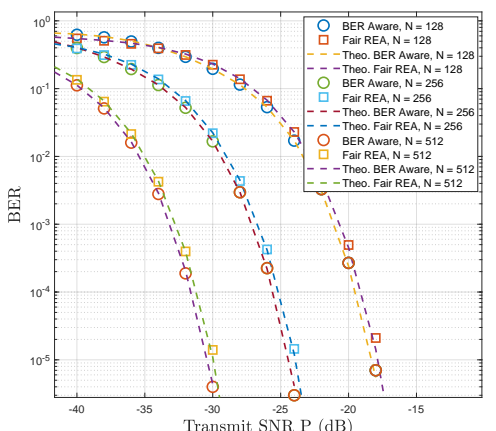

(c)

Figure 5. The simulation result of suitable ratio $r^{* *}$. (a) The result of $r^{* *}$ over different value of $P$ and $N$. (b) Comparison between proposed system and fair REA for different number of $N$. (c) The comparison of average users BER between BER aware and fair REA.

\section{REFERENCES}

[1] E. Basar, M. Di Renzo, J. De Rosny, M. Debbah, M. Alouini, and R. Zhang, "Wireless communications through reconfigurable intelligent surfaces," IEEE Access, vol. 7, pp. 116753-116773, 2019.

[2] Q. Wu and R. Zhang, "Towards smart and reconfigurable environment: Intelligent reflecting surface aided wireless network," IEEE Communications Magazine, vol. 58, no. 1, pp. 106-112, 2020.

[3] M. Di Renzo, K. Ntontin, J. Song, F. H. Danufane, X. Qian, F. Lazarakis, J. De Rosny, D.-T. Phan-Huy, O. Simeone, R. Zhang, M. Debbah, G. Lerosey, M. Fink, S. Tretyakov, and S. Shamai, "Reconfigurable intelligent surfaces vs. relaying: Differences, similarities, and performance comparison," IEEE Open Journal of the Communications Society, vol. 1, pp. 798-807, 2020.

[4] X. Liu, Y. Liu, Y. Chen, and H. V. Poor, "Ris enhanced massive nonorthogonal multiple access networks: Deployment and passive beamforming design," IEEE Journal on Selected Areas in Communications, vol. 39, no. 4, pp. 1057-1071, 2021.

[5] T. Hou, Y. Liu, Z. Song, X. Sun, Y. Chen, and L. Hanzo, "Reconfigurable intelligent surface aided NOMA networks," IEEE Journal on Selected Areas in Communications, pp. 1-1, 2020.

[6] L. Dai, B. Wang, Z. Ding, Z. Wang, S. Chen, and L. Hanzo, "A survey of non-orthogonal multiple access for $5 \mathrm{~g}$," IEEE Communications Surveys Tutorials, vol. 20, no. 3, pp. 2294-2323, 2018.

[7] Y. Saito, Y. Kishiyama, A. Benjebbour, T. Nakamura, A. Li, and K. Higuchi, "Non-orthogonal multiple access (noma) for cellular future radio access," in 2013 IEEE 77th Vehicular Technology Conference (VTC Spring), 2013, pp. 1-5.

[8] S. M. R. Islam, N. Avazov, O. A. Dobre, and K. Kwak, "Power-domain non-orthogonal multiple access (NOMA) in 5G systems: Potentials and challenges," IEEE Communications Surveys Tutorials, vol. 19, no. 2, pp. 721-742, Secondquarter 2017.

[9] Z. Wei, L. Yang, D. W. K. Ng, J. Yuan, and L. Hanzo, "On the performance gain of noma over oma in uplink communication systems," IEEE Transactions on Communications, vol. 68, no. 1, pp. 536-568, 2020.

[10] Z. Ding, Z. Yang, P. Fan, and H. V. Poor, "On the performance of non-orthogonal multiple access in $5 \mathrm{~g}$ systems with randomly deployed users," IEEE Signal Processing Letters, vol. 21, no. 12, pp. 1501-1505, 2014.

[11] X. Yue, Z. Qin, Y. Liu, S. Kang, and Y. Chen, "A unified framework for non-orthogonal multiple access," IEEE Transactions on Communications, vol. 66, no. 11, pp. 5346-5359, 2018.

[12] G. Yang, X. Xu, and Y.-C. Liang, "Intelligent reflecting surface assisted non-orthogonal multiple access," in 2020 IEEE Wireless Communications and Networking Conference (WCNC), 2020, pp. 1-6.

[13] M. Zhang, M. Chen, Z. Yang, H. Asgari, and M. Shikh-Bahaei, "Joint user clustering and passive beamforming for downlink noma system with reconfigurable intelligent surface," in 2020 IEEE 31st Annual International Symposium on Personal, Indoor and Mobile Radio Communications, 2020, pp. 1-6.

[14] Z. Ding and H. Vincent Poor, "A simple design of irs-noma transmission," IEEE Communications Letters, vol. 24, no. 5, pp. 1119-1123, 2020.
[15] X. Liu, Y. Liu, Y. Chen, and H. V. Poor, "Ris enhanced massive nonorthogonal multiple access networks: Deployment and passive beamforming design," IEEE Journal on Selected Areas in Communications, vol. 39, no. 4, pp. 1057-1071, 2021.

[16] Y. Li, M. Jiang, Q. Zhang, and J. Qin, "Joint beamforming design in multi-cluster miso noma reconfigurable intelligent surface-aided downlink communication networks," IEEE Transactions on Communications, vol. 69, no. 1, pp. 664-674, 2021.

[17] T. Hou, Y. Liu, Z. Song, X. Sun, and Y. Chen, "Mimo-noma networks relying on reconfigurable intelligent surface: A signal cancellation-based design," IEEE Transactions on Communications, vol. 68, no. 11, pp. 6932-6944, 2020.

[18] M. Elhattab, M.-A. Arfaoui, C. Assi, and A. Ghrayeb, "Reconfigurable intelligent surface assisted coordinated multipoint in downlink noma networks," IEEE Communications Letters, vol. 25, no. 2, pp. 632-636, 2021.

[19] Z. Ding, R. Schober, and H. V. Poor, "On the impact of phase shifting designs on irs-noma," IEEE Wireless Communications Letters, vol. 9, no. 10 , pp. 1596-1600, 2020.

[20] G. Yang, X. Xu, Y.-C. Liang, and M. D. Renzo, "Reconfigurable intelligent surface-assisted non-orthogonal multiple access," IEEE Transactions on Wireless Communications, vol. 20, no. 5, pp. 3137-3151, 2021.

[21] V. C. Thirumavalavan and T. S. Jayaraman, "Ber analysis of reconfigurable intelligent surface assisted downlink power domain NOMA system," in 2020 International Conference on COMmunication Systems NETworkS (COMSNETS), 2020, pp. 519-522.

[22] J. Zuo, Y. Liu, Z. Qin, and N. Al-Dhahir, "Resource allocation in intelligent reflecting surface assisted noma systems," IEEE Transactions on Communications, vol. 68, no. 11, pp. 7170-7183, 2020.

[23] A. Khaleel and E. Basar, "A novel noma solution with ris partitioning," IEEE Journal of Selected Topics in Signal Processing, pp. 1-1, 2021.

[24] M. Alam and Q. Zhang, "Qos-aware noma with sequence block compressed sensing multiuser detection," in 2019 IEEE Wireless Communications and Networking Conference (WCNC), 2019, pp. 1-6.

[25] C. Tepedelenlioglu, A. Abdi, and G. Giannakis, "The ricean k factor: estimation and performance analysis," IEEE Transactions on Wireless Communications, vol. 2, no. 4, pp. 799-810, 2003.

[26] M. Viswanathan and V. Mathuranathan, Wireless Communication Systems in Matlab: (Black \& White Edition). Independently Published, 2018. [Online]. Available: https://books.google.co.kr/books? id=zry5uwEACAAJ

[27] Fundamentals of Random Variables and Random Processes for Simulation. Boston, MA: Springer US, 2000, pp. 289-370. [Online]. Available: https://doi.org/10.1007/0-306-46971-5_6

[28] Proakis, Digital Communications 5th Edition. McGraw Hill, 2007.

[29] F. Kara and H. Kaya, "Performance analysis of SSK-NOMA," IEEE Transactions on Vehicular Technology, vol. 68, no. 7, pp. 6231-6242, 2019.

[30] J. Hu and N. Beaulieu, "Accurate closed-form approximations to ricean sum distributions and densities," IEEE Communications Letters, vol. 9, no. 2, pp. 133-135, 2005.

[31] S. Aja-Fernández and G. Vegas-Sánchez-Ferrero, "Statistical noise mod- 
els for mri," Springer International Publishing Switzerland, p. 275-291, 2016.

[32] A. Annamalai, C. Tellambura, and V. Bhargava, "Equal-gain diversity receiver performance in wireless channels," IEEE Transactions on Communications, vol. 48, no. 10, pp. 1732-1745, 2000.

[33] N. Beaulieu and X. Dong, "Level crossing rate and average fade duration of mrc and egc diversity in ricean fading," IEEE Transactions on Communications, vol. 51, no. 5, pp. 722-726, 2003.

[34] W. R. B. M. Schwartz and S. Stein, Communication systems and techniques. McGraw Hill, 1966.

[35] T. F. Coleman and Y. Li, "An interior trust region approach for nonlinear minimization subject to bounds," SIAM J. Optim., vol. 6, pp. 418-445, 1996.

[36] M. K. Simon and M.-S. Alouini, Digital communication over fading channels. John Wiley \& Sons, 2005, vol. 95.

[37] H. Zhang, B. Di, L. Song, and Z. Han, "Reconfigurable intelligent surfaces assisted communications with limited phase shifts: How many phase shifts are enough?" IEEE Transactions on Vehicular Technology, vol. 69, no. 4, pp. 4498-4502, 2020.

[38] A. Zappone, M. Di Renzo, X. Xi, and M. Debbah, "On the optimal number of reflecting elements for reconfigurable intelligent surfaces," IEEE Wireless Communications Letters, vol. 10, no. 3, pp. 464-468, 2021.

[39] M. B. Shahab and S. Y. Shin, "User pairing and power allocation for non-orthogonal multiple access: Capacity maximization under data reliability constraints," Physical Communication, vol. 30, pp. 132-144, 2018. [Online]. Available: https://www.sciencedirect.com/ science/article/pii/S1874490717305050

[40] S. Özyurt and O. Kucur, "Quadrature noma: A low-complexity multiple access technique with coordinate interleaving," IEEE Wireless Communications Letters, vol. 9, no. 9, pp. 1452-1456, 2020. 